\section{Active Oxygen}

THe light of the night sky has excited increased attention since the end of the War; from the practically minded student of rockets and from astronomers, as well as from students of the upper atmosphere. The astronomer is particularly interested because of the effect of this radiation on his observations. To the student of the upper atmosphere, this radiation has held out a promise of information concerning the composition of the upper air, as well as concerning fundamental processes there. Because of the difficult problem presented by spectroscopy of the light of the night sky, there are still many unidentified radiations in its spectrum, as well as inadequately studied regions. In every review of the contributions of spectroscopy to the study of the upper atmosphere, attention is directed to the desirability of further laboratory investigations of the spectra of $\mathrm{O}_{2}$ and $\mathrm{N}_{2}$, and the desirability of obtaining emission spectra of ozone and the oxides of nitrogen. It has been suggested that a search along these lines may yield a satisfactory identification of the strong ultra-violet band at $\lambda 3556 \mathrm{~A}$.

These facts, and some recent observations by Stebbins, Whitford and Swings ${ }^{1}$ at Mt. Wilson on one hand, and by R. Herman, L. Herman and J. Gauzit ${ }^{2}$ at the Lyons Observatory on the infra-red spectrum of the night sky on the other, led me to an extended study of the auroral afterglow in the infra-red. A particularly good nitrogen auroral afterglow at very low pressures was studied, and in time the intensity of the afterglow went down considerably, because of the decrease in pressure which resulted from the clean-up of the nitrogen. It was found that under these conditions the addition of relatively small quantities of oxygen restored the intensity of the afterglow in a very effective manner. This suggested that increasing the amount of oxygen might lead to interesting results, and this was done. It should be mentioned first that the neutral oxygen molecule does not readily show an emission spectrum. A part of the main absorption system observed by Schumann has been observed in emission by Runge, and hence the name SchumannRunge bands for these famous ultra-violet bands. Hopfield reported an emission spectrum in the region below $\lambda 2218 \mathrm{~A}$. consisting of a single progression. Other band systems, much too few in number and all consisting of transitions from the ground state to low-lying metastable states, have been observed in absorption in gaseous and liquid oxygen. The most famous of these are the so-called atmospheric bands, $A^{1} \Sigma \leftarrow X^{3} \Sigma$, observed in atmospheric absorption (especially the solar spectrum), and also observed in the laboratory by absorption through pure oxygen. It is this system which has now been observed for the first time in emission in the afterglow in oxygen, which resulted from the above procedure.

The name 'active oxygen' will be used to identify, this phenomenon, just as the name 'active nitrogen' was given to the similar phenomenon in nitrogen.

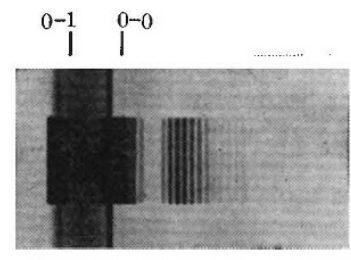

SPECTRUM OF ACTIVE OXYGEN
The spectrum of the afterglow of active oxygen which is reproduced shows two members of the atmospheric system of oxygen, which are readily identified as $0-0$ and $0-1$ by comparing them with the first positive system of nitrogen which is superposed. Using hypersensitized Eastman $I N$ plates, it is easy to obtain a good spectrogram in a time equal to that necessary for the nitrogen afterglow. It is quite likely that further studies of this afterglow will aid in the study of the physics of the upper atmosphere.

University of California,

Los Angeles, California.

Feb. 14

1 Stebbins, Whitford and Swings, Astrophys. J., 101, 39 (1945).

${ }^{2}$ Herman, R., Herman, L., and Gauzit, J., Nature, 156, 114 (1945).

\section{Structure of 'Terylene'}

Arising from the recent communication by Prof. W. T. Astbury and Mr. C. J. Brown ${ }^{1}$ concerning the structure of 'Terylene', we wish to place on record the earliest investigation of its structure by the X-ray method. The results recently reported by Astbury and Brown from work done in 1944 are in general agreement with those which we obtained some two years earlier.

Early in 1942, the Controller of Chemical Research, Ministry of Supply, learned of the work of Mr. J. R. Whinfield and Dr. J. T. Dickson (of the Calico Printers' Association, Ltd.) on polyethylene tere. phthalate ${ }^{2}$, and requested the Chemical Research Laboratory, D.S.I.R., to assist in its further develop. ment. As a result, almost the first step taken was to arrange for the National Physical Laboratory to carry out an X-ray examination of various 'Terylene' samples supplied by Mr. Whinfield and Dr. Dickson; this work was completed by July 7, 1942.

The granular powder produced by cooling a nitrobenzene solution of 'Terylene' gave the pattern shown in Fig. 1. The well-defined system of rings, corresponding to lattice-spacings of $10 \cdot 5,5 \cdot 4,5 \cdot 0,4 \cdot 1$, 3.9 and $3.4 \times 10^{-8} \mathrm{~cm}$., indicates that the material is definitely crystalline in the sense that neighbouring molecular chains bear a regular spatial relation to each other.

Slow cooling of molten 'Terylene' produces an opaque material which gave the pattern shown in Fig. 2. This is identical with Fig. 1, though the rings are somewhat sharper. The material has therefore the same structure, and the greater sharpness may be due to a larger particle size.

When molten 'Terylene' is shock-cooled, a translucent product is obtained; this gave the pattern shown in Fig. 3, which contains two diffuse haloes. The inner halo corresponds to the inner ring (spacing $10.5 \mathrm{~A}$.) of the previous forms. The outer halo corresponds to the outer group of diffraction rings of the previous forms; the rings have diffused together to form a broad halo. The molecular structure is therefore a heavily distorted form of the regular structure shown by the previously described crystalline forms.

Shock-cooled filaments may be cold-drawn into 'Terylene' fibres, and these gave the pattern shown in Fig. 4. This shows that very pronounced orientation of the molecules along the length of the fibre has taken place, the orientation being complete to within about $5^{\circ}$. The orientation is indicated by the con- 